\title{
Posteriori Error Estimation Method for Flexibility-Based Component Mode Synthesis
}

\author{
Jin-Gyun Kim* \\ Korea Institute of Machinery and Materials, Daejeon 305-343, Republic of Korea \\ and \\ Phill-Seung Lee \\ Korea Advanced Institute of Science and Technology, Daejeon 305-701, Republic of Korea
}

DOI: $\underline{10.2514 / 1 . J 053473}$

\begin{abstract}
The objective of this paper is to develop a simple and robust error estimation method for the flexibility-based component mode synthesis method. The flexibility-based component mode synthesis method is known to provide reliable and improved performance compared to other component mode synthesis methods. However, it is not possible to estimate errors in finite element models reduced by the flexibility-based component mode synthesis method yet. In this study, an a posteriori method is developed to accurately estimate relative errors in individual eigenvalues approximated by the flexibility-based component mode synthesis method without knowing the exact eigenvalues. The excellent performance of this error estimation method is demonstrated through various numerical examples.
\end{abstract}

\begin{tabular}{ll} 
& \multicolumn{1}{c}{ Nomenclature } \\
$B$ & $=$ interface Boolean matrix \\
$F$ & $=$ flexibility matrix \\
$f$ & $=$ force vector \\
$K$ & $=$ stiffness matrix \\
$L$ & $=$ assembly Boolean matrix \\
$M$ & $=$ mass matrix \\
$N$ & $=$ number of degrees of freedom or modes \\
$\boldsymbol{q}$ & $=$ generalized coordinate vector \\
$R$ & $=$ rigid-body mode shape matrix \\
$t$ & $=$ time variable \\
$\boldsymbol{u}$ & $=$ displacement vector \\
$\boldsymbol{\alpha}$ & $=$ generalized coordinate vector of rigid-body modes \\
$\delta$ & $=$ error term \\
$\eta$ & $=$ error estimator \\
$\lambda$ & $=$ eigenvalue \\
$\lambda_{\ell}$ & $=$ localized Lagrange multiplier vector \\
$\xi$ & $=$ relative eigenvalue error \\
$\Phi$ & $=$ eigenvector matrix \\
$\boldsymbol{\varphi}$ & $=$ eigenvector \\
$\omega$ & $=$ natural or forcing frequency
\end{tabular}

Subscripts

$b=$ interface boundary

$d=$ dominant term

$g=$ global structure

$p=$ partitioned structure

$q=$ deformable mode

$r \quad=$ residual term

$s \quad=$ substructure

$\alpha=$ rigid-body mode

\section{Introduction}

I STRUCTURAL engineering, the size of structural models has become larger and larger. It is a challenging problem to efficiently handle such large structural models. Component mode synthesis (CMS) is one of the popular reduced-order modeling techniques for the finite element models. CMS is also an efficient method to solve large eigenvalue problems in structural dynamics. In the 1960s, Hurty [1] and Guyan [2] first proposed the fundamental concept of CMS methods; since then, various CMS methods have been developed (see, e.g., [3-14]). The CMS methods are well summarized in [15].

In the $\mathrm{CM}$ S methods, the key to success is mainly due to the fact that a large original structural model is partitioned into small substructural models that can be easily handled. Then, instead of the large (nonpartitioned) global structural model, we deal with the reduced model constructed by dominant substructural modes. Therefore, CMS methods can dramatically reduce computational cost and match parallel computations fairly well [16].

The reliability of the reduced model can be assessed by errors in its approximated global eigenvalues. The eigenvalue errors also can measure the accuracy of the approximated eigensolutions when CMS methods are used to calculate the solutions of large eigenvalue problems. Therefore, it is very important to estimate accurately the errors when CMS methods are used. Basically, it is difficult to estimate the eigenvalue errors when the exact eigenvalues are unknown.

In an effort to handle this issue, Bourquin [17] developed an error bound derived from the exact global eigensolutions. Extending the error bound proposed by Yang et al. [18], Elssel and Voss [19] estimated relative eigenvalue errors for the Craig and Bampton $(\overline{\mathrm{CB}})$ and automated multilevel substructuring (AMLS) methods. Jakobsson and Larson [20] developed an a posteriori error estimation method of the approximated eigensolutions. In spite of the considerable efforts, the previous error estimation methods still provide qualitative tendency rather than meaningful quantity in eigenvalue errors. The methods were developed and tested only for the CB and AMLS methods.

To construct more reliable and accurate reduced models, Park and Park proposed the flexibility based component mode synthesis (FCMS) method [11]. However, it is not possible to estimate the reliability of models reduced by the F-CMS method. The objective of this study is to develop an a posteriori method that can accurately estimate individual eigenvalue errors for the F-CMS method. To derive the error estimator, the global exact eigensolutions are decomposed into approximated and error parts and the approximated eigenvectors are spanned by the exact eigenvectors. The decomposed eigensolutions are substituted into the global (original) eigenvalue problem and, analyzing resulting expanded terms, the relative 
eigenvalue error for the F-CMS method is obtained. The derivation procedure shows that the proposed error estimator can approximate the relative eigenvalue errors. Of course, the exact global eigenvalues are unknown in this error estimation method.

In the following sections, we first briefly review the F-CMS method in Sec. II. The fundamental concept and mathematical derivation of the proposed error estimation method are presented in Sec. III. In Sec. IV, its performance is tested through well-established numerical examples. The conclusions are given in Sec. $\underline{\text {. }}$

\section{Flexibility-Based Component Mode Synthesis}

The flexibility-based component mode synthesis method has been developed using the localized Lagrange multipliers $\lambda_{\ell}[11,21,22]$. Figures $1 \mathrm{a}$ and $1 \mathrm{~b}$ show a global structure $\Omega$ modeled by the finite element discretization and the structure partitioned into two substructures: $\Omega_{1}$ and $\Omega_{2} \quad\left(\Omega=\Omega_{1} \cup \Omega_{2}\right.$ and $\left.\Gamma=\Omega_{1} \cap \Omega_{2}\right)$, respectively. The two substructures are connected at the interface boundary $\Gamma$ with the constraint conditions using $\lambda_{\ell}$. Unlike the classical Lagrange multipliers, the localized Lagrange multipliers enable us to have the independent interface boundary degrees of freedom (DOFs) denoted by $\boldsymbol{u}_{b}$. Then, the substructures are individually connected with $\boldsymbol{u}_{b}$, as shown in Fig. 1c. The subscript $b$ refers to the interface boundary.

\section{A. Partitioned Formulation}

The displacement-based discrete energy functional $\Pi\left(\boldsymbol{u}_{g}\right)$ of the global structure is

$$
\Pi\left(\boldsymbol{u}_{g}\right)=\boldsymbol{u}_{g}^{T}\left(\frac{1}{2} K_{g} \boldsymbol{u}_{g}-\boldsymbol{f}_{g}+M_{g} \ddot{\boldsymbol{u}}_{g}\right), \quad M_{g}=L^{T} M_{s} L, \quad K_{g}=L^{T} K_{s} L
$$

where $M_{g}$ and $K_{g}$ are the global mass and stiffness matrices, respectively; $\boldsymbol{u}_{g}$ is the global displacement vector; and $\boldsymbol{f}_{g}$ is the global force vector. $M_{s}$ and $K_{s}$ are the partitioned block diagonal mass and stiffness matrices that consist of substructural mass and stiffness matrices, respectively. The subscripts $g$ and $s$ denote the global structure and substructures, respectively. The global structure and partitioned substructures are related by an assembly Boolean matrix $L$.

Then, $\delta \Pi\left(\boldsymbol{u}_{g}\right)$ yields the linear dynamics equations of the global structure

$$
M_{g} \ddot{\boldsymbol{u}}_{g}+K_{g} \boldsymbol{u}_{g}=\boldsymbol{f}_{g}
$$

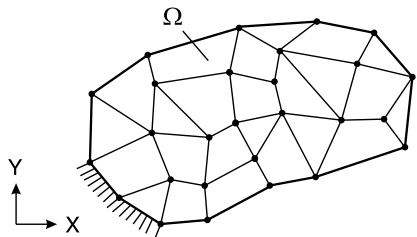

a)

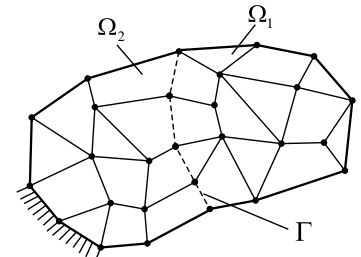

b)

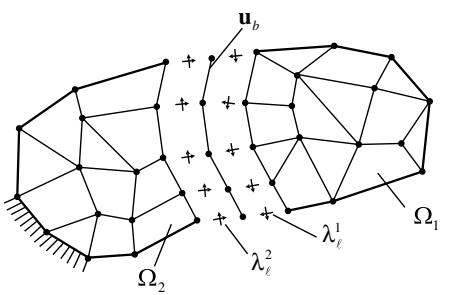

c)

Fig. 1 Global and partitioned structural models and interface handling in the F-CMS method: a) global (nonpartitioned) structure; b) partitioned structure; and c) interface boundary treatment.
To obtain the eigenvalues and eigenvectors of the global structure from Eq. (2), the following eigenvalue problem is solved:

$$
K_{g}\left(\boldsymbol{\varphi}_{g}\right)_{i}=\lambda_{i} M_{g}\left(\boldsymbol{\varphi}_{g}\right)_{i}, \quad i=1,2, \ldots, N_{g} \quad \text { with } \quad \boldsymbol{u}_{g}=\Phi_{g} \boldsymbol{q}_{g}
$$

where $\lambda_{i}$ and $\left(\varphi_{g}\right)_{i}$ are the $i$ th eigenvalue and eigenvector directly calculated from the global eigenvalue problem, respectively, and these are regarded as the exact global eigensolutions. Note that $\lambda_{i}$ is the square of the $i$ th natural frequency $\omega^{2}$ in structural dynamics. $N_{g}$ is the number of DOFs in the global structure. $\Phi_{g}$ and $\boldsymbol{q}_{g}$ are the global eigenvector matrix and generalized coordinate vector, respectively.

Using the localized Lagrange multipliers, $\Pi\left(\boldsymbol{u}_{g}\right)$ can be redefined as the sum of the substructural and constraint energy functionals

$$
\Pi\left(\boldsymbol{u}_{s}, \lambda_{\ell}, \boldsymbol{u}_{b}\right)=\boldsymbol{u}_{s}^{T}\left(\frac{1}{2} K_{s} \boldsymbol{u}_{s}-\boldsymbol{f}_{s}+M_{s} \ddot{\boldsymbol{u}}_{s}\right)+\lambda_{\ell}\left(B^{T} \boldsymbol{u}_{s}-L_{b} \boldsymbol{u}_{b}\right)
$$

where $\boldsymbol{u}_{s}$ and $\boldsymbol{f}_{s}$ are the partitioned displacement and force vectors of the substructures, respectively; and $B$ denotes the interface Boolean matrix. $L_{b}$ is obtained by condensing the rows with zero entries of $B^{T} L$ (see, e.g., [11]).

Here, the partitioned displacement vector $\boldsymbol{u}_{s}$ is decomposed by deformable and rigid-body modes

$$
\boldsymbol{u}_{s}=\Phi_{s} \boldsymbol{q}_{s}+R_{s} \boldsymbol{\alpha}_{s}
$$

where $\Phi_{s}$ and $R_{s}$ are the eigenvector matrices of the deformable and rigid-body modes, respectively. The corresponding generalized coordinate vectors are $\boldsymbol{q}_{s}$ and $\boldsymbol{\alpha}_{s}$. The block diagonal matrices $\Phi_{s}$ and $R_{s}$ are calculated from the substructural eigenvalue problems.

Then, Eq. (4) can be expressed by the energy functional of four variables $\Pi\left(\boldsymbol{q}_{s}, \boldsymbol{\alpha}_{s}, \lambda_{\ell}, \boldsymbol{u}_{b}\right)$, which yields

$$
\begin{gathered}
{\left[M_{p} \frac{\mathrm{d}^{2}}{\mathrm{~d} t^{2}}+K_{p}\right] \boldsymbol{u}_{p}=\boldsymbol{f}_{p}} \\
M_{p} \frac{\mathrm{d}^{2}}{\mathrm{~d} t^{2}}+K_{p}=\left[\begin{array}{cccc}
\Lambda_{s}+I_{q} \frac{\mathrm{d}^{2}}{\mathrm{~d} t^{2}} & 0 & \Phi_{s}^{T} B & 0 \\
0 & I_{\alpha} \frac{\mathrm{d}^{2}}{\mathrm{~d} t^{2}} & R_{b}^{T} & 0 \\
B^{T} \Phi_{s} & R_{b} & 0 & -L_{b} \\
0 & 0 & -L_{b}^{T} & 0
\end{array}\right], \\
\boldsymbol{u}_{p}=\left[\begin{array}{c}
\boldsymbol{q}_{s} \\
\boldsymbol{\alpha}_{s} \\
\lambda_{\ell} \\
\boldsymbol{u}_{b}
\end{array}\right], \quad \boldsymbol{f}_{p}=\left[\begin{array}{c}
\Phi_{s}^{T} \boldsymbol{f}_{s} \\
R_{s}^{T} \boldsymbol{f}_{s} \\
0 \\
0
\end{array}\right], \\
I_{q}=\Phi_{s}^{T} M_{s} \Phi_{s}, \quad \Lambda_{s}=\Phi_{s}^{T} K_{s} \Phi_{s}, \\
I_{\alpha}=R_{s}^{T} M_{s} R_{s},
\end{gathered}
$$

where $M_{p}$ and $K_{p}$ are the partitioned mass and stiffness matrices, respectively. The partitioned displacement and force vectors are $\boldsymbol{u}_{p}$ and $\boldsymbol{f}_{p}$, respectively. Subscript $p$ denotes the partitioned structure, and $t$ denotes the time variable. Note that Eq. (6) contains all the deformable modes $\Phi_{s}$ and rigid-body modes $R_{s}$.

\section{B. Reduction Procedure}

The eigenvector matrix of the deformable substructural modes $\Phi_{s}$ can be divided into the dominant and residual parts

$$
\Phi_{s} \boldsymbol{q}_{s}=\Phi_{d} \boldsymbol{q}_{d}+\Phi_{r} \boldsymbol{q}_{r}
$$


where subscripts $d$ and $r$ denote the dominant and residual terms, respectively. $\Phi_{d}$ and $\Phi_{r}$ are the dominant and residual substructural eigenvector matrices, respectively; and $\boldsymbol{q}_{d}$ and $\boldsymbol{q}_{r}$ are the corresponding generalized coordinate vectors. $\Phi_{d}$ consists of $N_{d}$ dominant substructural eigenvectors $\left(N_{d}=N_{d}^{(1)}+N_{d}^{(2)}+\ldots+N_{d}^{\left(N_{s}\right)}\right)$, and the remaining substructural eigenvectors regarded as the residual modes are put into $\Phi_{r} . N_{s}$ denotes the number of substructures.

Substituting Eq. (7) into Eq. (6) and performing the Gauss elimination on $\boldsymbol{q}_{r}$, we obtain the following condensed equations:

$$
\begin{aligned}
& {\left[\begin{array}{cccc}
\hat{\Lambda}_{d} & 0 & \Phi_{d b}^{T} & 0 \\
0 & I_{\alpha} \frac{\mathrm{d}^{2}}{\mathrm{~d} t^{2}} & R_{b}^{T} & 0 \\
\Phi_{d b} & R_{b} & -\hat{F}_{r b} & -L_{b} \\
0 & 0 & -L_{b}^{T} & 0
\end{array}\right]\left[\begin{array}{c}
\boldsymbol{q}_{d} \\
\boldsymbol{\alpha}_{s} \\
\lambda_{\ell} \\
\boldsymbol{u}_{b}
\end{array}\right]=\left[\begin{array}{c}
\Phi_{d}^{T} \boldsymbol{f}_{s} \\
R_{s}^{T} \boldsymbol{f}_{s} \\
-B^{T} \hat{F}_{r} \boldsymbol{f}_{s} \\
0
\end{array}\right],} \\
& \hat{\Lambda}_{d}=\Lambda_{d}+\frac{\mathrm{d}^{2}}{\mathrm{~d} t^{2}} I_{d}, \quad \Lambda_{d}=\Phi_{d}^{T} K_{s} \Phi_{d}, \quad I_{d}=\Phi_{d}^{T} M_{s} \Phi_{d}, \\
& \Phi_{d b}=B^{T} \Phi_{d}, \quad \hat{F}_{r b}=B^{T} \hat{F}_{r} B, \quad \hat{F}_{r}=\Phi_{r}\left[\Lambda_{r}+\frac{\mathrm{d}^{2}}{\mathrm{~d} t^{2}} I_{r}\right]^{-1} \Phi_{r}^{T}
\end{aligned}
$$

in which $\hat{F}_{r}$ is the dynamic residual flexibility, and $\hat{F}_{r b}$ is its interface boundary part, respectively.

We then invoke the harmonic response $\left(\mathrm{d}^{2} / \mathrm{d} t^{2}=-\omega^{2}\right) . \hat{F}_{r}$ and $\hat{F}_{r b}$ are expressed as functions of $\omega^{2}$, and these yield an intractable eigenvalue problem. To treat the transcendental nature of the interface residual flexibility $\hat{F}_{r}$, we approximate

$$
\begin{aligned}
& \hat{F}_{r}=\Phi_{r}\left[\Lambda_{r}-\omega^{2} I_{r}\right]^{-1} \Phi_{r}^{T} \approx F_{r s}+\omega^{2} F_{r m}, \\
& F_{r s}=\Phi_{r} \Lambda_{r}^{-1} \Phi_{r}^{T}, \quad F_{r m}=\Phi_{r} \Lambda_{r}^{-2} \Phi_{r}^{T}
\end{aligned}
$$

where $F_{r s}$ and $F_{r m}$ are the static and dynamic parts of $\hat{F}_{r}$, and these can be indirectly calculated using the full flexibility and the inverse of the stiffness matrix $K_{s}$. Since $K_{s}$ contains rigid-body modes in the F-CMS method, it is not invertible. Therefore, the pseudoinverse denoted by the superscript + is used as

$$
\begin{gathered}
F_{r s}=M_{s}^{-1 / 2}\left[M_{s}^{-1 / 2} K_{s} M_{s}^{-1 / 2}\right]^{+} M_{s}^{-1 / 2}-\Phi_{d} \Lambda_{d}^{-1} \Phi_{d}^{T} \\
F_{r m}=M_{s}^{-1 / 2}\left[M_{s}^{-1 / 2} K_{s} M_{s}^{-1 / 2}\right]^{+2} M_{s}^{-1 / 2}-\Phi_{d} \Lambda_{d}^{-2} \Phi_{d}^{T}
\end{gathered}
$$

Then, the interface residual flexibility $\hat{F}_{r b}$ can be also defined:

$$
\hat{F}_{r b} \approx F_{r b s}+\omega^{2} F_{r b m}, \quad F_{r b s}=B^{T} F_{r s} B, \quad F_{r b m}=B^{T} F_{r m} B
$$

Substituting Eq. (11) into Eq. (8) with the free vibration condition $(f=0)$, we obtain the final reduced eigenvalue problem

$$
\begin{gathered}
\bar{K}_{p}\left(\overline{\boldsymbol{\varphi}}_{p}\right)_{i}=\bar{\lambda}_{i} \bar{M}_{p}\left(\overline{\boldsymbol{\varphi}}_{p}\right)_{i}, \quad i=1,2, \ldots, N_{p} \\
\bar{K}_{p}=\left[\begin{array}{cccc}
\Lambda_{d} & 0 & \Phi_{d b}^{T} & 0 \\
0 & 0 & R_{b}^{T} & 0 \\
\Phi_{d b} & R_{b} & -F_{r b s} & -L_{b} \\
0 & 0 & -L_{b}^{T} & 0
\end{array}\right], \quad \bar{M}_{p}=\left[\begin{array}{cccc}
I_{d} & 0 & 0 & 0 \\
0 & I_{\alpha} & 0 & 0 \\
0 & 0 & F_{r b m} & 0 \\
0 & 0 & 0 & 0
\end{array}\right], \\
\bar{\Phi}_{p}=\left[\begin{array}{l}
\bar{\Phi}_{q_{d}} \\
\bar{\Phi}_{\alpha_{s}} \\
\bar{\Phi}_{\lambda_{\ell}} \\
\bar{\Phi}_{u_{b}}
\end{array}\right]
\end{gathered}
$$

where $\bar{M}_{p}$ and $\bar{K}_{p}$ are the reduced partitioned mass and stiffness matrices, respectively; and $\bar{\lambda}_{i}$ and $\left(\overline{\boldsymbol{\varphi}}_{p}\right)_{i}$ are the eigensolutions of the reduced model, respectively. Note that an overbar denotes approximated quantities.

From the third row in Eq. (12), the approximated global eigenvector $\left(\overline{\boldsymbol{\varphi}}_{g}\right)_{i}$ is computed by

$$
\left(\boldsymbol{\varphi}_{g}\right)_{i} \approx\left(\overline{\boldsymbol{\varphi}}_{g}\right)_{i}=\Phi_{d}\left(\overline{\boldsymbol{\varphi}}_{q_{d}}\right)_{i}+R_{s}\left(\overline{\boldsymbol{\varphi}}_{\alpha_{s}}\right)_{i}-\hat{F}_{r} B\left(\overline{\boldsymbol{\varphi}}_{\lambda_{\ell}}\right)_{i}
$$

In the F-CMS method, the residual modes are not simply truncated but transformed into the residual flexibility as shown in Eq. (9). In contrast, in stiffness-based CMS methods such as the CB and AMLS methods, those residual modes are truncated. This fact leads to the improvement of accuracy in the F-CMS method. Note that the approximated global eigenvector $\left(\overline{\boldsymbol{\varphi}}_{g}\right)_{i}$ in Eq. (13) does not satisfy the mass orthonormality for the global model as

$$
\left(\overline{\boldsymbol{\varphi}}_{g}\right)_{i}^{T} M_{g}\left(\overline{\boldsymbol{\varphi}}_{g}\right)_{j} \neq \delta_{i j}
$$

where $\delta_{i j}$ is Kronecker delta ( $\delta_{i j}=1$ if $i=j$; otherwise, $\delta_{i j}=0$ ). Using this feature, it is possible to develop an error estimator for the F-CMS method. However, the feature cannot be directly employed for the CB method, which is one of the most popular CMS methods. This issue will be presented in the following section.

\section{Error Estimation Method}

\section{A. Derivation Procedure}

As mentioned in the Introduction (Sec. I), it is very important to estimate the eigenvalue error in CMS methods. In so doing, the $i$ th relative eigenvalue error $\xi_{i}$ is generally used:

$$
\xi_{i}=\frac{\bar{\lambda}_{i}-\lambda_{i}}{\lambda_{i}}=\frac{\bar{\lambda}_{i}}{\lambda_{i}}-1
$$

To obtain the exact relative eigenvalue error, the exact global eigenvalue $\lambda_{i}$ calculated from the global eigenvalue problem in Eq. (3) is a prerequisite. In this study, we focus on how to estimate the relative eigenvalue error when the exact global eigenvalue $\lambda_{i}$ is unknown.

Here, we first define the relation between the exact and approximated global eigensolutions. The exact global eigenvalue $\lambda_{i}$ and eigenvector $\left(\varphi_{g}\right)_{i}$ satisfy the global eigenvalue problem in Eq. (3). The eigensolutions $\left[\bar{\lambda}_{i},\left(\overline{\boldsymbol{\varphi}}_{p}\right)_{i}\right]$ of the reduced model are obtained from the reduced eigenvalue problem in Eq. (12), and the approximated global eigenvector $\left(\overline{\boldsymbol{\varphi}}_{g}\right)_{i}$ is calculated from Eq. (13) using $\left(\overline{\boldsymbol{\varphi}}_{p}\right)_{i}$.

The exact global eigensolutions are expressed by the approximated global eigensolutions and the error terms $\delta \lambda_{i}$ and $\left(\delta \varphi_{g}\right)_{i}$ :

$$
\lambda_{i}=\bar{\lambda}_{i}+\delta \lambda_{i}
$$

$$
\left(\boldsymbol{\varphi}_{g}\right)_{i}=\left(\overline{\boldsymbol{\varphi}}_{g}\right)_{i}+\left(\delta \boldsymbol{\varphi}_{g}\right)_{i}
$$

Since the exact global eigenvectors are linearly independent, the approximated global eigenvector $\left(\overline{\boldsymbol{\varphi}}_{g}\right)_{i}$ can be represented by a linear combination of the exact global eigenvectors

$$
\left(\overline{\boldsymbol{\varphi}}_{g}\right)_{i}=\sum_{k=1}^{N_{g}} \alpha_{k}\left(\boldsymbol{\varphi}_{g}\right)_{k}
$$

where the coefficient $\alpha_{k}$ can be calculated by premultiplying $\left(\overline{\boldsymbol{\varphi}}_{g}\right)_{k}^{T} M_{g}$. 
Using Eq. (17) in Eq. (16b), the eigenvector error $\left(\delta \varphi_{g}\right)_{i}$ can be represented by $\alpha_{k}$ and $\left(\varphi_{g}{\overline{)_{k}}}_{\text {: }}\right.$ :

$$
\begin{aligned}
& \left(\delta \boldsymbol{\varphi}_{g}\right)_{i}=-\left[\alpha_{1}\left(\boldsymbol{\varphi}_{g}\right)_{1}+\alpha_{2}\left(\boldsymbol{\varphi}_{g}\right)_{2}+\cdots+\left(\alpha_{i}-1\right)\left(\boldsymbol{\varphi}_{g}\right)_{i}+\cdots\right. \\
& \left.\quad+\alpha_{N_{g}}\left(\boldsymbol{\varphi}_{g}\right)_{N_{g}}\right]
\end{aligned}
$$

It is important to note that, as we increase the number of substructural modes retained in the reduced model, the approximated global eigenvector $\left(\overline{\boldsymbol{\varphi}}_{g}\right)_{i}$ becomes close to the exact global eigenvector $\left(\varphi_{g}\right)_{i}$. When the approximated global eigenvector is close enough to the exact global eigenvector, we can assume that

$\alpha_{i} \approx 1, \quad\left|\alpha_{i}\right| \gg\left|\alpha_{i}-1\right|, \quad\left|\alpha_{1}\right|, \quad\left|\alpha_{2}\right| \cdots\left|\alpha_{i-1}\right|$,

$\left|\alpha_{i+1}\right|, \cdots,\left|\alpha_{N_{g}}\right|, \quad$ and $\quad \bar{\lambda}_{i} \approx \lambda_{i}$

Note that, when the $i$ th exact and approximated global eigenvectors $\left[\left(\boldsymbol{\varphi}_{g}\right)_{i}=\left(\overline{\boldsymbol{\varphi}}_{g}\right)_{i}\right]$ are identical, the coefficient $\alpha_{i}=1$ and other coefficients are zero.

Since $\lambda_{i}$ and $\left(\varphi_{g}\right)_{i}$ are the solutions of the global eigenvalue problem in Eq. (3), the following equations are satisfied:

$$
\frac{1}{\lambda_{i}}\left(\boldsymbol{\varphi}_{g}\right)_{i}^{T} K_{g}\left(\boldsymbol{\varphi}_{g}\right)_{i}=\left(\boldsymbol{\varphi}_{g}\right)_{i}^{T} M_{g}\left(\boldsymbol{\varphi}_{g}\right)_{i}
$$

and

$$
\left(\boldsymbol{\varphi}_{g}\right)_{i}^{T} M_{g}\left(\boldsymbol{\varphi}_{g}\right)_{i}=1, \quad\left(\boldsymbol{\varphi}_{g}\right)_{i}^{T} K_{g}\left(\boldsymbol{\varphi}_{g}\right)_{i}=\lambda_{i}
$$

Using Eq. (16b) in Eq. (20), we obtain

$$
\begin{aligned}
\frac{1}{\lambda_{i}} & {\left[\left(\overline{\boldsymbol{\varphi}}_{g}\right)_{i}+\left(\delta \boldsymbol{\varphi}_{g}\right)_{i}\right]^{T} K_{g}\left[\left(\overline{\boldsymbol{\varphi}}_{g}\right)_{i}+\left(\delta \boldsymbol{\varphi}_{g}\right)_{i}\right] } \\
& =\left[\left(\overline{\boldsymbol{\varphi}}_{g}\right)_{i}+\left(\delta \boldsymbol{\varphi}_{g}\right)_{i}\right]^{T} M_{g}\left[\left(\overline{\boldsymbol{\varphi}}_{g}\right)_{i}+\left(\delta \boldsymbol{\varphi}_{g}\right)_{i}\right]
\end{aligned}
$$

Then, the left-hand side of Eq. (22) can be rewritten:

$$
\begin{aligned}
\frac{1}{\lambda_{i}} & {\left[\left(\overline{\boldsymbol{\varphi}}_{g}\right)_{i}+\left(\delta \boldsymbol{\varphi}_{g}\right)_{i}\right]^{T} K_{g}\left[\left(\overline{\boldsymbol{\varphi}}_{g}\right)_{i}+\left(\delta \boldsymbol{\varphi}_{g}\right)_{i}\right] } \\
& =\frac{1}{\lambda_{i}}\left(\overline{\boldsymbol{\varphi}}_{g}\right)_{i}^{T} K_{g}\left(\overline{\boldsymbol{\varphi}}_{g}\right)_{i}+\frac{2}{\lambda_{i}}\left(\delta \boldsymbol{\varphi}_{g}\right)_{i}^{T} K_{g}\left(\overline{\boldsymbol{\varphi}}_{g}\right)_{i}+\frac{1}{\lambda_{i}}\left(\delta \boldsymbol{\varphi}_{g}\right)_{i}^{T} K_{g}\left(\delta \boldsymbol{\varphi}_{g}\right)_{i}
\end{aligned}
$$

Using Eq. (18) in the second and third terms of Eq. (23), we have

$$
\begin{gathered}
\frac{2}{\lambda_{i}}\left(\delta \boldsymbol{\varphi}_{g}\right)_{i}^{T} K_{g}\left(\overline{\boldsymbol{\varphi}}_{g}\right)_{i}=-2\left(\alpha_{i}-1\right)-2\left(\alpha_{i}-1\right)^{2}-2 \sum_{\substack{k=1 \\
k \neq i}}^{N_{g}} \alpha_{k}^{2} \frac{\lambda_{k}}{\lambda_{i}} \\
\frac{1}{\lambda_{i}}\left(\delta \boldsymbol{\varphi}_{g}\right)_{i}^{T} K_{g}\left(\delta \boldsymbol{\varphi}_{g}\right)_{i}=\left(\alpha_{i}-1\right)^{2}+\sum_{\substack{k=1 \\
k \neq i}}^{N_{g}} \alpha_{k}^{2} \frac{\lambda_{k}}{\lambda_{i}}
\end{gathered}
$$

$$
\begin{aligned}
\frac{1}{\lambda_{i}} & {\left[\left(\overline{\boldsymbol{\varphi}}_{g}\right)_{i}+\left(\delta \boldsymbol{\varphi}_{g}\right)_{i}\right]^{T} K_{g}\left[\left(\overline{\boldsymbol{\varphi}}_{g}\right)_{i}+\left(\delta \boldsymbol{\varphi}_{g}\right)_{i}\right] } \\
& =\frac{1}{\lambda_{i}}\left(\overline{\boldsymbol{\varphi}}_{g}\right)_{i}^{T} K_{g}\left(\overline{\boldsymbol{\varphi}}_{g}\right)_{i}-2\left(\alpha_{i}-1\right)-\left(\alpha_{i}-1\right)^{2}-\sum_{\substack{k=1 \\
k \neq i}}^{N_{g}} \alpha_{k}^{2} \frac{\lambda_{k}}{\lambda_{i}}
\end{aligned}
$$

Similarly, we can expand the right-hand side of Eq. (22):

$$
\begin{aligned}
& {\left[\left(\overline{\boldsymbol{\varphi}}_{g}\right)_{i}+\left(\delta \boldsymbol{\varphi}_{g}\right)_{i}\right]^{T} M_{g}\left[\left(\overline{\boldsymbol{\varphi}}_{g}\right)_{i}+\left(\delta \boldsymbol{\varphi}_{g}\right)_{i}\right]} \\
& \quad=\left(\overline{\boldsymbol{\varphi}}_{g}\right)_{i}^{T} M_{g}\left(\overline{\boldsymbol{\varphi}}_{g}\right)_{i}+2\left(\delta \boldsymbol{\varphi}_{g}\right)_{i}^{T} M_{g}\left(\overline{\boldsymbol{\varphi}}_{g}\right)_{i}+\left(\delta \boldsymbol{\varphi}_{g}\right)_{i}^{T} M_{g}\left(\delta \boldsymbol{\varphi}_{g}\right)_{i} \\
& \quad=\left(\overline{\boldsymbol{\varphi}}_{g}\right)_{i}^{T} \boldsymbol{M}_{g}\left(\overline{\boldsymbol{\varphi}}_{g}\right)_{i}-2\left(\alpha_{i}-1\right)-\left(\alpha_{i}-1\right)^{2}-\sum_{\substack{k=1 \\
k \neq i}}^{N_{g}} \alpha_{k}^{2}
\end{aligned}
$$

Using Eqs. (25) and (26) in Eq. (22), we obtain

$$
\frac{1}{\lambda_{i}}\left(\overline{\boldsymbol{\varphi}}_{g}\right)_{i}^{T} K_{g}\left(\overline{\boldsymbol{\varphi}}_{g}\right)_{i}=\left(\overline{\boldsymbol{\varphi}}_{g}\right)_{i}^{T} M_{g}\left(\overline{\boldsymbol{\varphi}}_{g}\right)_{i}+\left[\sum_{\substack{k=1 \\ k \neq i}}^{N_{g}} \alpha_{k}^{2}\left(\frac{\lambda_{k}}{\lambda_{i}}-1\right)\right]
$$

Note that the leading-order terms $2\left(\alpha_{i}-1\right)$ in Eqs. (25) and (26) are canceled.

Here, we assume that the global stiffness matrix $K_{g}$ can be divided into the approximated global stiffness matrix and its error

$$
K_{g}=\bar{K}_{g}+\delta K_{g}
$$

in which $\delta K_{g}$ is error in the global stiffness matrix, and $\bar{K}_{g}$ is the approximated global stiffness matrix that satisfies

$$
\left(\overline{\boldsymbol{\varphi}}_{g}\right)_{k}^{T} \bar{K}_{g}\left(\overline{\boldsymbol{\varphi}}_{g}\right)_{k}=\bar{\lambda}_{k} \quad \text { for } k=1,2, \ldots, N_{p}
$$

Using Eqs. (28) and (29) in Eq. (27), we obtain

$$
\frac{\bar{\lambda}_{i}}{\lambda_{i}}=\left(\overline{\boldsymbol{\varphi}}_{g}\right)_{i}^{T} M_{g}\left(\overline{\boldsymbol{\varphi}}_{g}\right)_{i}+\left[\sum_{\substack{k=1 \\ k \neq i}}^{N_{g}} \alpha_{k}^{2}\left(\frac{\lambda_{k}}{\lambda_{i}}-1\right)\right]-\frac{1}{\lambda_{i}}\left(\overline{\boldsymbol{\varphi}}_{g}\right)_{i}^{T} \delta K_{g}\left(\overline{\boldsymbol{\varphi}}_{g}\right)_{i}
$$

In the right-hand side of Eq. (30), the second and third terms are much smaller than the first term under the assumption in Eq. (19). Neglecting the small terms, the following equation is given:

$$
\frac{\bar{\lambda}_{i}}{\lambda_{i}} \approx\left(\overline{\boldsymbol{\varphi}}_{g}\right)_{i}^{T} M_{g}\left(\overline{\boldsymbol{\varphi}}_{g}\right)_{i}
$$

and, subtracting one from the left- and right-hand sides of Eq. (31), we obtain

$$
\frac{\bar{\lambda}_{i}}{\lambda_{i}}-1 \approx\left(\overline{\boldsymbol{\varphi}}_{g}\right)_{i}^{T} M_{g}\left(\overline{\boldsymbol{\varphi}}_{g}\right)_{i}-1
$$

where the left-hand side is the relative error in the $i$ th eigenvalue in Eq. (15), and the right-hand side is the error in mass orthonormality for the $i$ th approximated global eigenvector $\left(\overline{\boldsymbol{\varphi}}_{g}\right)_{i}$ with global mass matrix $M_{g}$.

Using the relation in Eq. (32), we can define the error estimator $\eta_{i}$ for the $i$ th approximated eigenvalue in the F-CMS method 


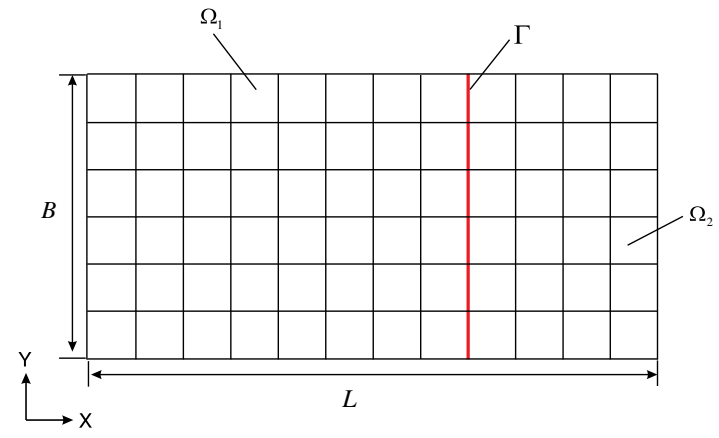

Fig. 2 Rectangular plate problem.

$$
\eta_{i}=\left(\overline{\boldsymbol{\varphi}}_{g}\right)_{i}^{T} M_{g}\left(\overline{\boldsymbol{\varphi}}_{g}\right)_{i}-1
$$

with

$$
\left(\overline{\boldsymbol{\varphi}}_{g}\right)_{i}=\Phi_{d}\left(\overline{\boldsymbol{\varphi}}_{q_{d}}\right)_{i}+R_{s}\left(\overline{\boldsymbol{\varphi}}_{\alpha_{s}}\right)_{i}-\left[F_{r s}+\bar{\lambda}_{i} F_{r m}\right] B\left(\overline{\boldsymbol{\varphi}}_{\lambda_{\ell}}\right)_{i}
$$

It is important to note that, in order to obtain Eq. (34), the unknown $\omega^{2}$ contained in $\hat{F}_{r}$ [see Eq. (9)] is substituted by the $\overline{i \text { th }}$ approximated eigenvalue $\bar{\lambda}_{i}$ calculated from the reduced eigenvalue problem. Consequently, the preceding derivation procedure shows that the error estimator $\eta_{i}$ can be used to approximate the exact relative eigenvalue error $\xi_{i}$ in Eq. $(15)\left(\eta_{i} \approx \xi_{i}\right)$. We also note that the computational cost for the error estimator $\eta_{i}$ is not high because only simple additions and multiplications of known matrices are required.

Mathematically, the proposed error estimator $\eta_{i}$ is not defined as the upper or lower bound of errors. Recalling that the proposed error estimation method is valid under the assumptions in Eqs. (19) and (28), it is also important to investigate the accuracy of the error estimator $\eta_{i}$ depending on the number of retained dominant substructural modes. This is studied in numerical examples.

The proposed error estimator can be also employed to develop effective model reduction algorithms in combination with various mode selection methods $[21,23]$ and error estimator for other model reduction methods [24]. After determining the error tolerance for target global modes, we can iteratively select the retained substructural modes until the error tolerance is properly satisfied. Then, a small reduced model with a given accuracy can be effectively constructed.

\section{B. Feasibility for the Craig and Bampton Method}

Mathematically, the global eigenvector $\left(\boldsymbol{\varphi}_{g}\right)_{i}$ always satisfies the mass orthonormality for the global model as

$$
\left(\boldsymbol{\varphi}_{g}\right)_{i}^{T} M_{g}\left(\boldsymbol{\varphi}_{g}\right)_{j}=\delta_{i j}
$$

However, the approximated global eigenvector $\left(\overline{\boldsymbol{\varphi}}_{g}\right)_{i}$ is not the direct eigensolution for the global model; thus, $\left(\overline{\boldsymbol{\varphi}}_{g}\right)_{i}$ does not satisfy the mass orthonormality for the global model in general as in Eq. (14).

Table 1 Retained substructural mode numbers $N_{d}^{(k)}$ in the rectangular plate problem using the $\mathrm{CB}$ and F-CMS methods

\begin{tabular}{cccccccc}
\hline \hline & \multicolumn{3}{c}{$\mathrm{CB}$} & & \multicolumn{3}{c}{ F-CMS } \\
\cline { 2 - 4 } \cline { 6 - 8 } Numerical cases & $N_{d}^{(1)}$ & $N_{d}^{(2)}$ & $N_{d}$ & & $N_{d}^{(1)}$ & $N_{d}^{(2)}$ & $N_{d}$ \\
\hline Case 1 & 7 & 3 & 10 & & 7 & 3 & 10 \\
Case 2 & 13 & 7 & 20 & & 13 & 7 & 20 \\
\hline \hline
\end{tabular}

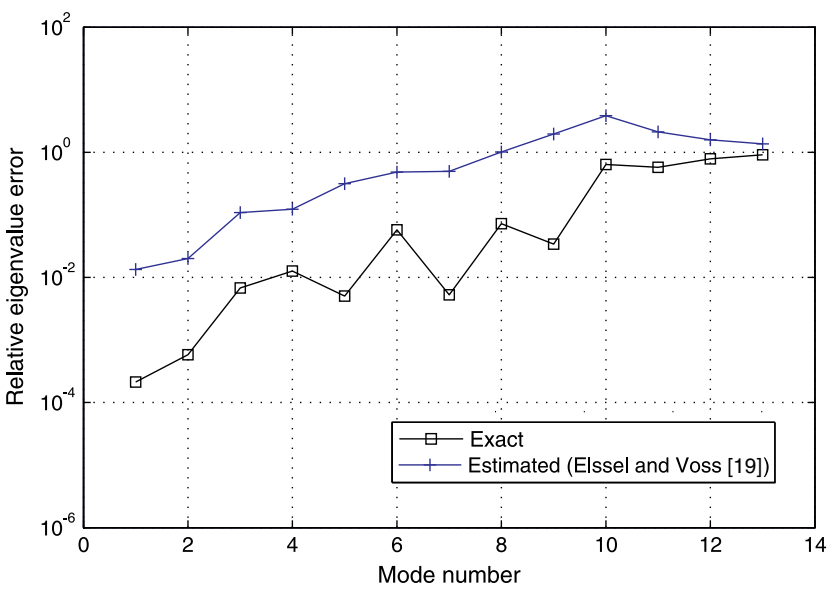

a)

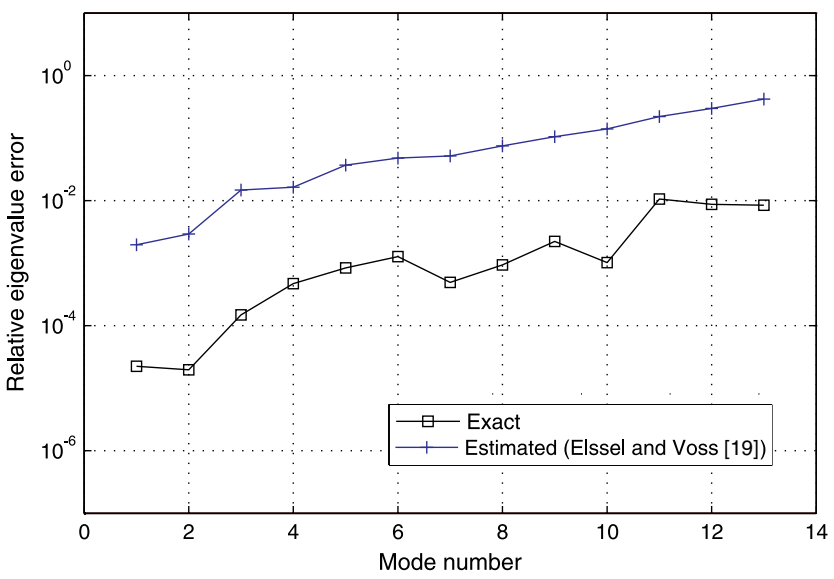

b)

Fig. 3 Exact and estimated relative eigenvalue errors in the rectangular plate problem using the CB method: a) $N_{d}=10$, and b) $N_{d}=20$.

As $\left(\overline{\boldsymbol{\varphi}}_{g}\right)_{i}$ becomes closer to $\left(\boldsymbol{\varphi}_{g}\right)_{i}$, Eq. (14) gets closer to $\delta_{i j}$. Using this feature, we derived an estimator $\eta_{i}$ of the relative eigenvalue errors for the F-CMS method in Sec. III.A.

Since the proposed error estimator $\overline{\eta_{i}}$ is derived from the general description of CMS methods, it may be applied for not only the FCMS method but also for other CMS methods. However, the present form of $\eta_{i}$ cannot be directly used for a type of CMS method like the CB method [3] . In the CB method, the transformation matrix $\bar{T}_{\mathrm{CB}}$ constructed by the dominant substructural modes is used to calculate the reduced matrices as

$$
\bar{M}_{p}=\bar{T}_{\mathrm{CB}}^{T} M_{g} \bar{T}_{\mathrm{CB}}, \quad \bar{K}_{p}=\bar{T}_{\mathrm{CB}}^{T} K_{g} \bar{T}_{\mathrm{CB}}
$$

And, using the same transformation matrix $\bar{T}_{\mathrm{CB}}$, the approximated global eigenvector $\left(\overline{\boldsymbol{\varphi}}_{g}\right)_{i}$ can be also obtained from the eigenvector $\left(\overline{\boldsymbol{\varphi}}_{p}\right)_{i}$ calculated in the reduced model

$$
\left(\overline{\boldsymbol{\varphi}}_{g}\right)_{i}=\bar{T}_{\mathrm{CB}}\left(\overline{\boldsymbol{\varphi}}_{p}\right)_{i}
$$

Using Eqs. (36) and (37) in the left-hand side of Eq. (14), the following relation is obtained:

$$
\left(\overline{\boldsymbol{\varphi}}_{g}\right)_{i}^{T} M_{g}\left(\overline{\boldsymbol{\varphi}}_{g}\right)_{j}=\left(\overline{\boldsymbol{\varphi}}_{p}\right)_{i}^{T} \bar{M}_{p}\left(\overline{\boldsymbol{\varphi}}_{p}\right)_{j}
$$

Since the eigenvector $\left(\overline{\boldsymbol{\varphi}}_{p}\right)_{i}$ is the eigensolution of the reduced model, it is easy to identify 


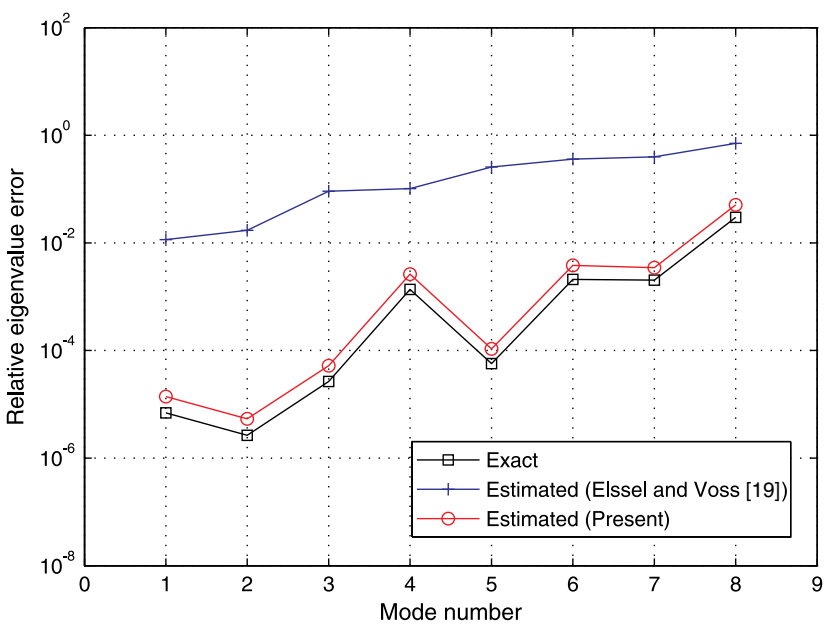

a)

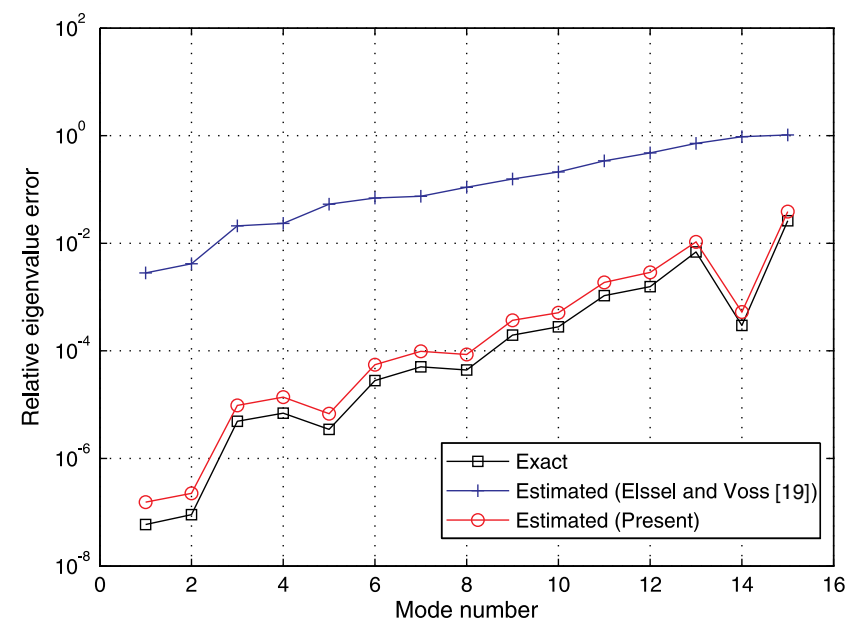

b)

Fig. 4 Exact and estimated relative eigenvalue errors in the rectangular plate problem using the F-CMS method: a) $N_{d}=10$, and b) $N_{d}=20$.

$$
\begin{aligned}
& \left(\overline{\boldsymbol{\varphi}}_{g}\right)_{i}^{T} M_{g}\left(\overline{\boldsymbol{\varphi}}_{g}\right)_{j}=\left(\overline{\boldsymbol{\varphi}}_{p}\right)_{i}^{T} \bar{M}_{p}\left(\overline{\boldsymbol{\varphi}}_{p}\right)_{j}=\delta_{i j} \\
& \quad \text { for } i \text { and } j=1,2, \ldots, N_{p}
\end{aligned}
$$

Therefore, in the CB method, the mass orthonormality of $\left(\overline{\boldsymbol{\varphi}}_{g}\right)_{i}$ for the global model is mathematically identical with the mass
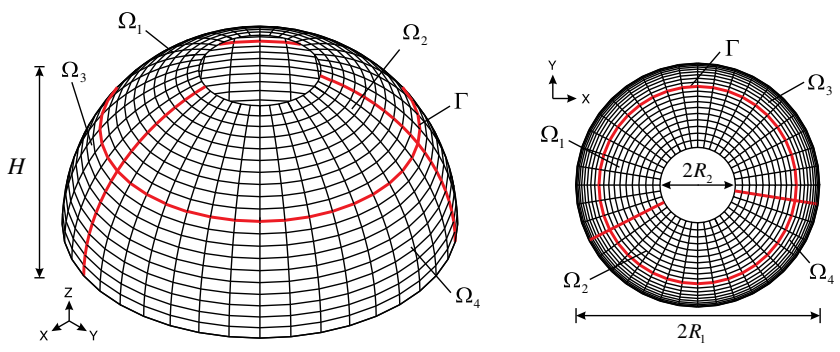

Fig. 5 Hemisphere shell problem.

orthonormality of $\left(\overline{\boldsymbol{\varphi}}_{p}\right)_{j}$ for the reduced model. For this reason, the proposed error estimator $\eta_{i}$ in Eq. (33) always gives zero in the CB method, and thus cannot be directly used for the $\mathrm{CB}$ method in its present form. However, the conceptual idea of the present error estimator can be used to develop an error estimator for the $\mathrm{CB}$ method; see [25].

\section{Numerical Examples}

In this section, we test the performance of the proposed error estimation method. For the finite element models, we use the fournode mixed interpolation of tensorial components (MITC) shell finite elements (see, e.g., [26-29]).

Here, we compare the proposed error estimation method with a previous error estimation method proposed by Elssel and Voss [19]. It was developed as an upper bound of the relative eigenvalue error

$$
\tau_{i}=\frac{\bar{\lambda}_{i}}{\left|\lambda_{r}-\bar{\lambda}_{i}\right|}
$$

where $\lambda_{r}$ is the smallest residual eigenvalue of substructures. Although this method was originally developed for the $\mathrm{CB}$ and AMLS methods based on the frequency cutoff mode selection method, it can also evaluate individual eigenvalue errors in the FCMS method with similar accuracy.

Table 3 Retained substructural mode numbers $N_{d}^{(k)}$ in the hemisphere shell problem using the F-CMS method

\begin{tabular}{|c|c|c|c|c|c|c|}
\hline \multirow[b]{2}{*}{ Mode number } & \multicolumn{3}{|c|}{$N_{d}=10$} & \multicolumn{3}{|c|}{$N_{d}=20$} \\
\hline & Exact & $\begin{array}{c}\text { Estimated } \\
\text { (Elssel and Voss [14]) }\end{array}$ & $\begin{array}{l}\text { Estimated } \\
\text { (present) }\end{array}$ & Exact & $\begin{array}{c}\text { Estimated } \\
\text { (Elssel and Voss [14]) }\end{array}$ & $\begin{array}{l}\text { Estimated } \\
\text { (present) }\end{array}$ \\
\hline 1 & $6.94762 \mathrm{E}-06$ & $1.14848 \mathrm{E}-02$ & $1.38687 \mathrm{E}-05$ & $5.89399 \mathrm{E}-08$ & $2.80305 E-03$ & $1.54027 \mathrm{E}-07$ \\
\hline 2 & $2.66257 \mathrm{E}-06$ & $1.71028 \mathrm{E}-02$ & $5.34686 \mathrm{E}-06$ & $9.04237 \mathrm{E}-08$ & $4.15680 \mathrm{E}-03$ & $2.24763 \mathrm{E}-07$ \\
\hline 3 & $2.65320 \mathrm{E}-05$ & $9.13660 \mathrm{E}-02$ & $5.22991 \mathrm{E}-05$ & $4.89581 \mathrm{E}-06$ & $2.10428 \mathrm{E}-02$ & $9.70832 \mathrm{E}-06$ \\
\hline 4 & $1.36731 \mathrm{E}-03$ & $1.02404 \mathrm{E}-01$ & $2.62666 \mathrm{E}-03$ & $6.93182 \mathrm{E}-06$ & $2.33709 E-02$ & $1.38077 \mathrm{E}-05$ \\
\hline 5 & $5.69427 \mathrm{E}-05$ & $2.57811 \mathrm{E}-01$ & $1.06987 \mathrm{E}-04$ & $3.46214 \mathrm{E}-06$ & $5.31378 \mathrm{E}-02$ & $6.80650 \mathrm{E}-06$ \\
\hline 6 & $2.08814 \mathrm{E}-03$ & $3.59515 \mathrm{E}-01$ & $3.83258 \mathrm{E}-03$ & $2.81479 \mathrm{E}-05$ & $6.67814 \mathrm{E}-01$ & $5.53600 \mathrm{E}-05$ \\
\hline 7 & $2.04114 \mathrm{E}-03$ & $3.96652 \mathrm{E}-01$ & $6.46290 \mathrm{E}-03$ & $5.05974 \mathrm{E}-05$ & $7.50112 E-02$ & $9.82232 \mathrm{E}-05$ \\
\hline 8 & $2.98671 \mathrm{E}-02$ & $7.07415 \mathrm{E}-01$ & $5.06769 \mathrm{E}-02$ & $4.41654 \mathrm{E}-05$ & $1.09932 \mathrm{E}-01$ & $8.57886 \mathrm{E}-05$ \\
\hline 9 & -- & -- & -- & $1.95870 \mathrm{E}-04$ & $1.56472 \mathrm{E}-01$ & $3.70223 E-04$ \\
\hline 10 & -- & -- & -- & $2.78901 E-04$ & $2.11385 \mathrm{E}-01$ & $5.12478 \mathrm{E}-04$ \\
\hline 11 & -- & -- & -- & $1.06043 E-03$ & $3.41084 \mathrm{E}-01$ & $1.87649 \mathrm{E}-03$ \\
\hline 12 & -- & -- & -- & $1.55768 \mathrm{E}-03$ & $4.77692 \mathrm{E}-01$ & $2.86479 \mathrm{E}-03$ \\
\hline 13 & -- & -- & -- & $6.88535 \mathrm{E}-03$ & $7.20722 \mathrm{E}-01$ & $1.05754 \mathrm{E}-02$ \\
\hline 14 & -- & -- & -- & $2.97041 \mathrm{E}-04$ & $9.57359 \mathrm{E}-01$ & $5.25239 \mathrm{E}-04$ \\
\hline 15 & -- & -- & -- & $2.61556 \mathrm{E}-02$ & $1.02923 \mathrm{E}+00$ & $3.87200 \mathrm{E}-02$ \\
\hline
\end{tabular}

\begin{tabular}{lccccc}
\hline \hline Numerical cases & $N_{d}^{(1)}$ & $N_{d}^{(2)}$ & $N_{d}^{(3)}$ & $N_{d}^{(4)}$ & $N_{d}$ \\
\hline Case 1 & 12 & 8 & 20 & 14 & 54 \\
Case 2 & 25 & 17 & 35 & 23 & 100 \\
\hline \hline
\end{tabular}

Table 2 Exact and estimated eigenvalue errors in the rectangular plate problem 


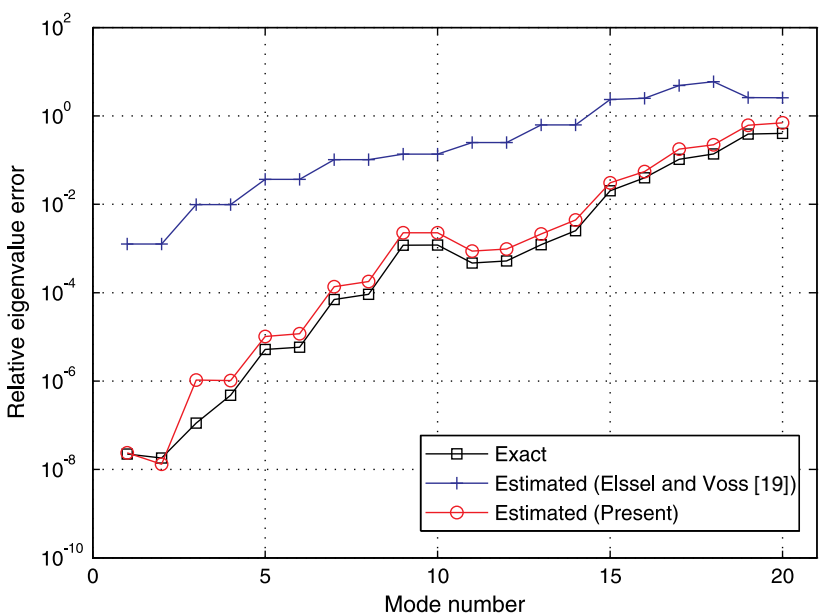

a)

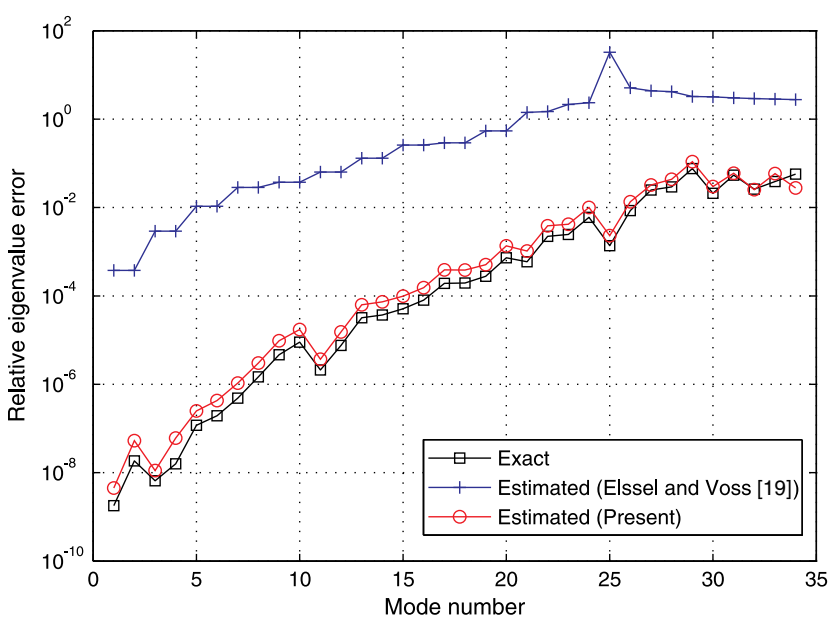

b)

Fig. 6 Exact and estimated relative eigenvalue errors in the hemisphere shell problem using the F-CMS method: a) $N_{d}=54$, and b) $N_{d}=100$.

\section{A. Rectangular Plate Problem}

Let us consider a rectangular plate with a free boundary; see Fig. 2 . The length $L$ is $0.6096 \mathrm{~m}$, the width $B$ is $0.3048 \mathrm{~m}$, and the thickness $h$ is $3.175 \times 10^{-3} \mathrm{~m}$. Young's modulus $E$ is $72 \mathrm{G} \mathrm{Pa}$, Poisson's ratio $\nu$ is 0.33 , and the density $\rho$ is $2796 \mathrm{~kg} / \mathrm{m}^{3}$. The plate is modeled by a $12 \times 6$ mesh of the four-node MITC shell finite elements, and the structural model is partitioned into two substructures.

Since the error estimation method by Elssel and Voss [19] in Eq. (40) was originally developed for the CB and AMLS methods, we first test its performance in the $\mathrm{CB}$ method. The frequency cutoff mode selection method is employed to select dominant substructural modes. Two numerical cases are considered for the number of retained substructural modes, and the numbers of the retained substructural modes are listed in Table $\underline{1}$. Figure $\underline{3}$ shows the exact relative eigenvalue errors $\xi_{i}$ and estimated relative eigenvalue errors $\tau_{i}$. The numerical results show that $\tau_{j}$ provides an upper bound for the exact relative eigenvalue error $\xi_{j}$, and the difference between $\tau_{j}$ and $\xi_{j}$ is about one or two digits on a log scale, as presented in [19].

Figure $\underline{4}$ presents the exact and estimated relative errors in eigenvalues when the F-CMS method is employed. To select the dominant substructural modes, we adopt the frequency cutoff mode selection method. The numbers of retained substructural modes are listed in Table 1 .

The performance of the error estimation method by Elssel and Voss [19] is similar in both the CB and F-CMS methods. The numerical results show the excellent performance of the proposed error estimation method for both numerical cases compared with the
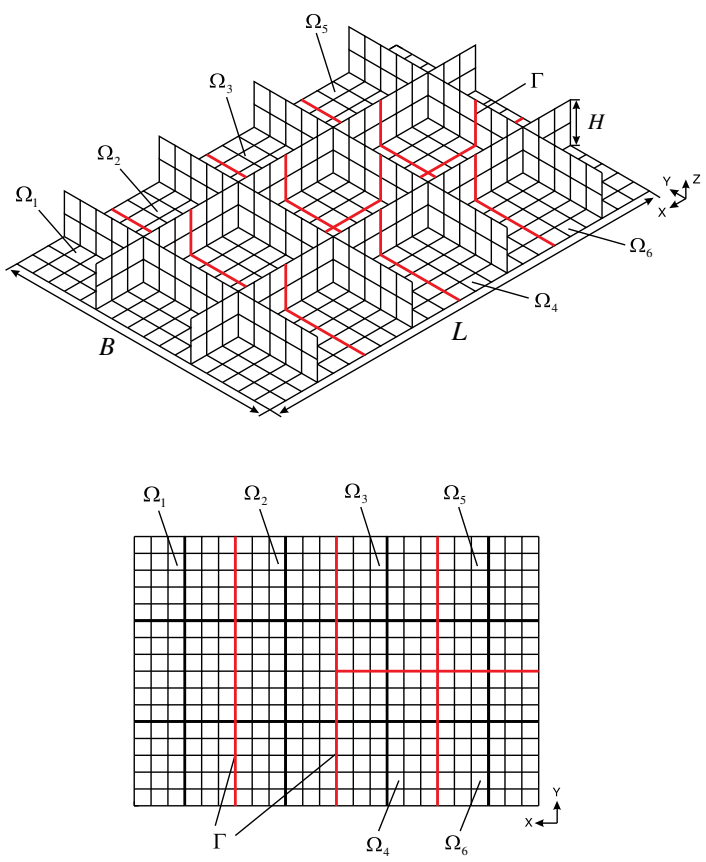

Fig. 7 Stiffened plate problem.

previous error estimation method. The proposed error estimation method provides an upper bound for the exact relative eigenvalue error in the problem considered here. The exact and estimated eigenvalues errors are given in Table 2.

It should be also noted that Elssel and Voss's method [19] is an a priori method that was developed for giving the upper bound of errors in the CB method. However, the proposed method is an a posteriori method focusing on accurately estimating the relative eigenvalue errors in the F-CMS method. Therefore, this difference should be considered when numerical results are investigated.

\section{B. Hemisphere Shell Problem}

Let us consider a hemisphere shell with a free boundary condition at both ends; see Fig. 5. The height $H$ is $3.084 \mathrm{~m}$, and the thickness $h$ is $0.05 \mathrm{~m}$. The radii $R_{1}$ and $R_{2}$ are 2 and $0.618 \mathrm{~m}$, respectively. Young's modulus $E$ is $69 \mathrm{G} \mathrm{Pa}$, Poisson's ratio $\nu$ is 0.35 , and the density $\rho$ is $2700 \mathrm{~kg} / \mathrm{m}^{3}$. For the finite element model, 20 and 40 four-node MITC shell finite elements are used in the axial and circumferential directions, respectively. The structure is partitioned into four substructures.

Using the F-CMS method, we consider two numerical cases for the number of retained substructural modes: 54 and 100 substructural modes, as listed in Table 3 . Figure 6 shows the excellent performance of the proposed error estimation method. As in the previous numerical example, the proposed error estimation acts as an upper bound for the exact relative eigenvalue error in general.

\section{Stiffened Plate Problem}

Here, the proposed error estimation method is used for a stiffened plate with a free boundary; see Fig. 7. The length $L$ is $4.8 \mathrm{~m}$, the width $B$ is $3.2 \mathrm{~m}$, and the thickness $h$ is $0.03 \mathrm{~m}$. The plate has two and four stiffeners in the longitudinal and transverse directions, respectively.

Table 4 Retained substructural mode numbers $N_{d}^{(k)}$ in the stiffened plate problem using the F-CMS method

\begin{tabular}{lccccccc}
\hline \hline Numerical cases & $N_{d}^{(1)}$ & $N_{d}^{(2)}$ & $N_{d}^{(3)}$ & $N_{d}^{(4)}$ & $N_{d}^{(5)}$ & $N_{d}^{(6)}$ & $N_{d}$ \\
\hline Case 1 & 8 & 8 & 4 & 4 & 4 & 4 & 32 \\
Case 2 & 12 & 12 & 7 & 7 & 7 & 7 & 52 \\
\hline \hline
\end{tabular}




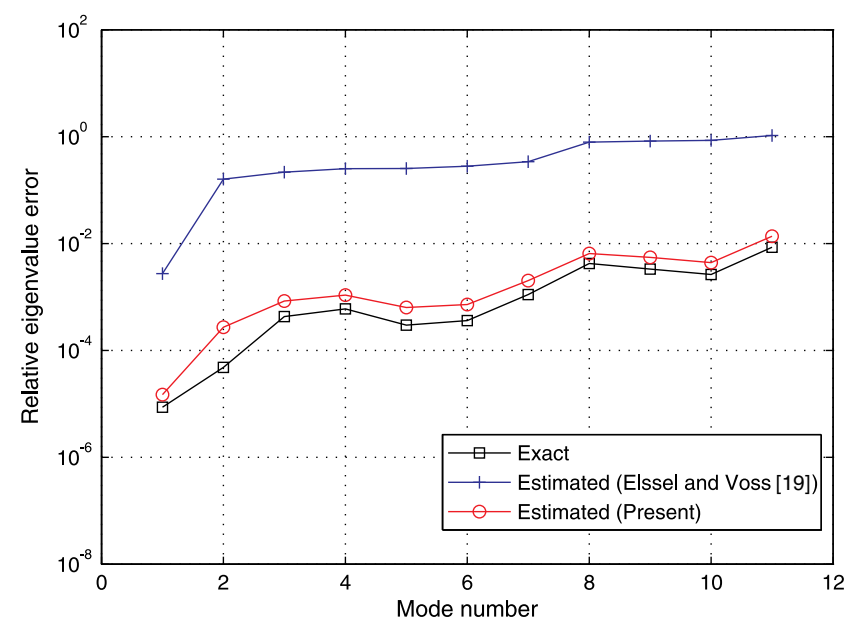

a)

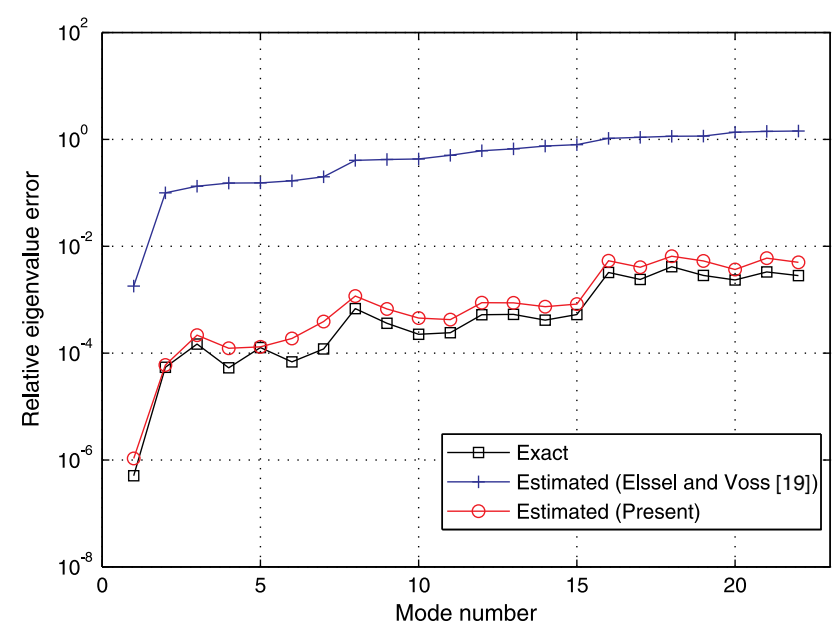

b)

Fig. 8 Exact and estimated relative eigenvalue errors in the stiffened plate problem using the F-CMS method: a) $N_{d}=32$, and b) $N_{d}=\mathbf{5 2}$.

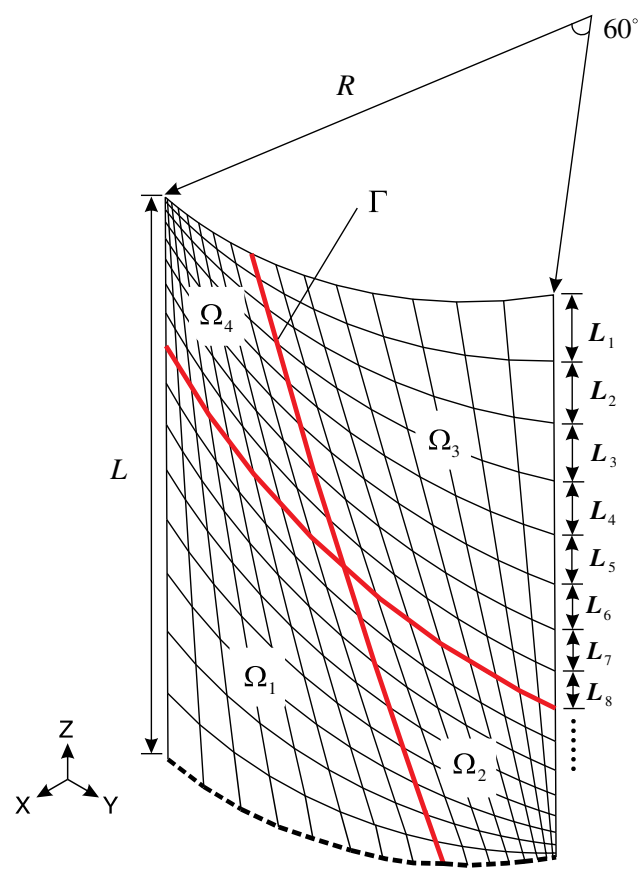

Fixed boundary

Fig. 9 Cylindrical panel problem with a distorted mesh.
Table 5 Retained substructural mode numbers $N_{d}^{(k)}$ in the cylindrical panel problem using the F-CMS method

\begin{tabular}{lrrrrr}
\hline \hline Numerical cases & $N_{d}^{(1)}$ & $N_{d}^{(2)}$ & $N_{d}^{(3)}$ & $N_{d}^{(4)}$ & $N_{d}$ \\
\hline Case 1 & 7 & 12 & 6 & 12 & 37 \\
Case 2 & 9 & 15 & 8 & 16 & 48 \\
\hline \hline
\end{tabular}

The stiffeners have a height of $H=0.5 \mathrm{~m}$ and a thickness of $h=0.03 \mathrm{~m}$. Young's modulus $E$ is $210 \mathrm{G} \mathrm{Pa}$, Poisson's ratio $\nu$ is 0.3 , and the density $\rho$ is $7850 \mathrm{~kg} / \mathrm{m}^{3}$. The plate is modeled by a $24 \times 16$ mesh of four-node MITC shell finite elements, and the longitudinal and transverse stiffeners are modeled by $24 \times 2$ and $16 \times 2$ meshes of four-node MITC shell finite elements, respectively. This stiffened plate is partitioned into six substructures.

In this problem, we consider two cases for the number of retained substructural modes: 32 and 52 substructural mode; see Table 4. As shown in Fig. $\underline{8}$, the proposed error estimation method can very accurately estimate the relative eigenvalue error in the reduced model constructed by the F-CMS method. Also, the method provides an upper bound rather than a lower bound in this problem.

\section{Cylindrical Panel Problem}

Let us consider a cylindrical panel problem, shown in Fig. 9. The panel structure is fixed along the bottom edge. The length $L$ is $0.8 \mathrm{~m}$, the radius is $R$ is $0.5 \mathrm{~m}$, and the thickness $h$ is $0.005 \mathrm{~m}$. Young's

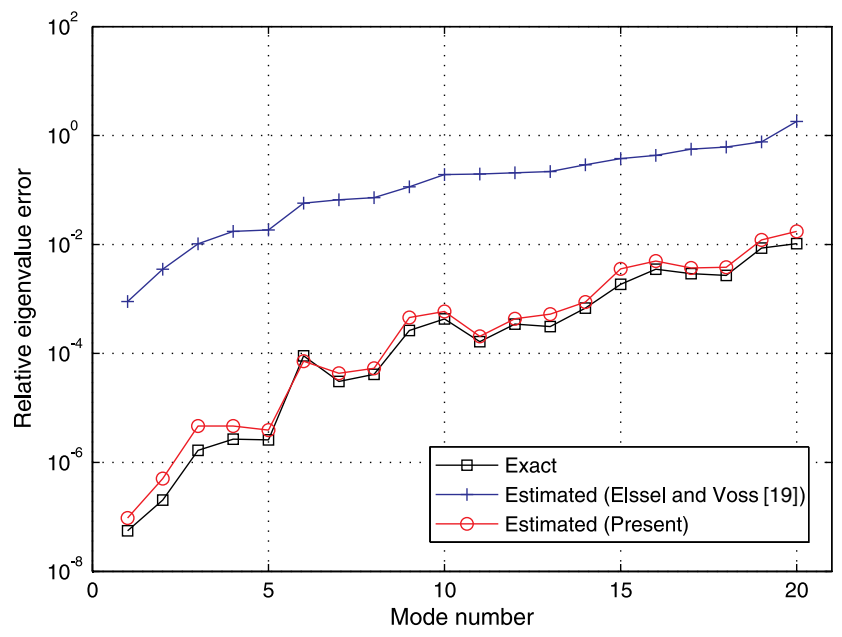

a)

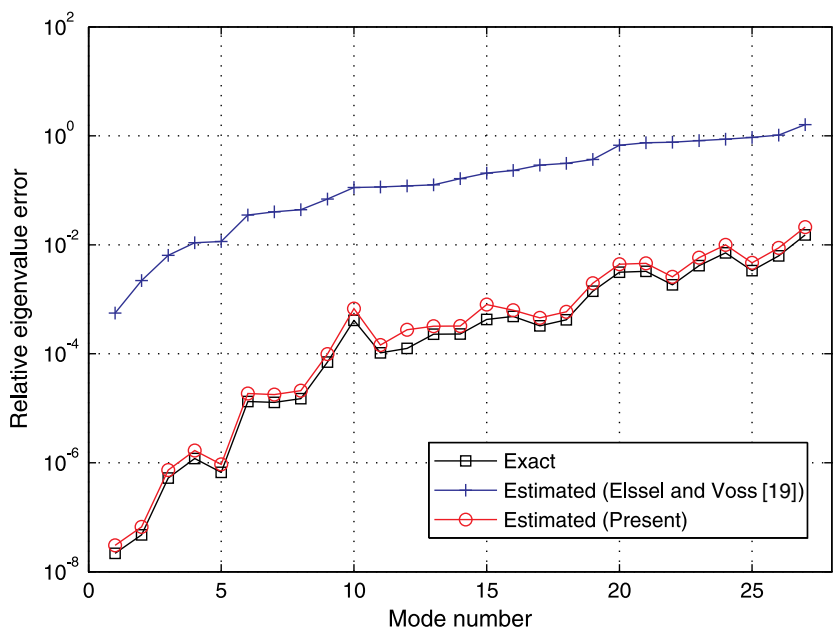

b)

Fig. 10 Exact and estimated relative eigenvalue errors in the cylindrical panel problem using the F-CMS method: a) $N_{d}=37$, and b) $N_{d}=48$. 
modulus $E$ is $69 \mathrm{G} \mathrm{Pa}$, Poisson's ratio $\nu$ is 0.35 , and the density $\rho$ is $2700 \mathrm{~kg} / \mathrm{m}^{3}$. We model the structure with a $16 \times 16$ distorted mesh of four-node shell finite elements, in which each edge is discretized in the following ratio: $L_{1}: L_{2}: L_{3}: \cdots L_{16}=16: 15: 14: \cdots 1$. The structural model is partitioned into four substructures. For two numerical cases, 37 and 48 substructural modes are selected; see Table 5. Figure 10 shows that the proposed error estimation method also performs well in a distorted mesh.

In this study, we considered only undamped structural models, but the proposed method can be also employed for model reductions of damped structural models [30].

\section{Conclusions}

In this paper, an accurate error estimation method was developed for the flexibility-based component mode synthesis method. This error estimation method provides relative errors in individual eigenvalues for the flexibility based component mode synthesis (F-CMS) method. Through four numerical examples, such as rectangular plate, hemisphere shell, stiffened plate, and cylindrical panel problems, the performance of the proposed error estimation method was demonstrated. The numerical results showed that the proposed error estimation method is consistently accurate. In most of the numerical cases considered in this study, the error estimator provides a tight upper bound for the exact relative eigenvalue error, even when the retained dominant substructural modes are relatively few.

It will be valuable in future works to apply the concept of the proposed error estimation method to other component mode synthesis methods $[10,12]$. It is noted that the proposed error estimation method can be adopted to develop effective model reduction algorithms in combination with various mode selection methods $[22,23]$. In addition, the error estimation method could be employed for the time-integration schemes with the F-CMS method.

\section{Acknowledgments}

This work was supported by the Human Resources Development Program (no. 20134030200300) of the Korea Institute of Energy Technology Evaluation and Planning funded by the Ministry of Trade, Industry and Energy; and the Basic Science Research Program through the National Research Foundation of Korea funded by the Ministry of Education, Science and Technology (no. 2014R1A1A1A05007219).

\section{References}

[1] Hurty, W., "Dynamic Analysis of Structural Systems Using Component Modes," AIAA Journal, Vol. 3, No. 4, 1965, pp. 678-685. doi: $10.2514 / 3.2947$

[2] Guyan, R., "Reduction of Stiffness and Mass Matrices," AIAA Journal, Vol. 3, No. 2, 1965, p. 380. doi: $10.2514 / 3.2874$

[3] Craig, R. R., and Bampton, M. C. C., "Coupling of Substructures for Dynamic Analysis," AIAA Journal, Vol. 6, No. 7, 1968, pp. 1313-1319. doi: $10.2514 / 3.4741$

[4] MacNeal, R. H., "Hybrid Method of Component Mode Synthesis," Computers and Structures, Vol. 1, No. 4, 1971, pp. 581-601. doi:10.1016/0045-7949(71)90031-9

[5] Benfield, W. A., and Hruda, R. F., "Vibration Analysis of Structures by Component Mode Substitution," AIAA Journal, Vol. 9, No. 7, 1971, pp. $1255-1261$. doi:10.2514/3.49936

[6] Rubin, S., "Improved Component-Mode Representation for Structural Dynamic Analysis," AIAA Journal, Vol. 13, No. 8, 1975, pp. 995-1006. doi: $10.2514 / 3.60497$

[7] Hintz, R. M., "Analytical Methods in Component Modal Synthesis," AIAA Journal, Vol. 13, No. 8, 1975, pp. 1007-1016. doi: $10.2514 / 3.60498$

[8] Shyu, W. H., Ma, Z. D., and Hulbert, G. M., "A New Component Mode Synthesis Method: Quasi-Static Mode Compensation," Finite Elements in Analysis and Design, Vol. 24, No. 4, 1997, pp. 271-281. doi:10.1016/S0168-874X(96)00066-2

[9] Shyu, W. H., Gu, J., Hulbert, G. M., and Ma, Z. D., "On the Use of Multiple Quasi-Static Mode Compensation Sets for Component Mode Synthesis of Complex Structures," Finite Elements in Analysis and
Design, Vol. 35, No. 2, 2000, pp. 119-140.

doi:10.1016/S0168-874X(99)00058-X

[10] Bennighof, J. K., and Lehoucq, R. B., "An Automated Multilevel Substructuring Method for Eigenspace Computation in Linear Elastodynamics," SIAM Journal on Scientific Computing, Vol. 25, No. 6, 2004, pp. 2084-2106. doi:10.1137/S1064827502400650

[11] Park, K. C., and Park, Y. H., "Partitioned Component Mode Synthesis via a Flexibility Approach," AIAA Journal, Vol. 42, No. 6, 2004, pp. 1236-1245. doi:10.2514/1.10423

[12] Rixen, D. J., "A Dual Craig-Bampton Method for Dynamic Substructuring," Journal of Computational and Applied Mathematics, Vol. 168, Nos. 1-2, 2004, pp. 383-391. doi:10.1016/j.cam.2003.12.014

[13] Kim, J. G., Boo, S. H., and Lee, P. S., "The Enhanced AMLS Method and Its Performance," Computer Methods in Applied Mechanics and Engineering, Vol. 287, April 2015, pp. 90-111. doi:10.1016/j.cma.2015.01.004

[14] Kim, J. G., and Lee, P. S., "An Enhanced Craig-Bampton Method," International Journal for Numerical Methods in Engineering, Vol. 103, No. 2, July 2015, pp. 79-93.

doi: $10.1002 / \mathrm{nme} .4880$

[15] Klerk, D. D., Rixen, D. J., and Voormeeren, S. N., "General Framework for Dynamic Substructuring: History, Review, and Classification of Techniques," AIAA Journal, Vol. 46, No. 5, 2008, pp. 1169-1181. doi:10.2514/1.33274

[16] Farhat, C., and Roux, F. X., "A Method of Finite Element Tearing and Interconnecting and Its Parallel Solution Algorithm," International Journal for Numerical Methods in Engineering, Vol. 32, No. 6, 1991, pp. 1205-1227. doi:10.1002/(ISSN)1097-0207

[17] Bourquin, F., "Analysis and Comparison of Several Component Mode Synthesis Methods on One Dimensional Domains," Numerische Mathematik, Vol. 58, No. 1, 1990, pp. 11-33. doi:10.1007/BF01385608

[18] Yang, C., Gao, W., Bai, Z., Li, X. S., Lee, L. Q., Husbands, P., and Ng, E., "An Algebraic Substructuring Method for Large-Scale Eigenvalue Calculation," SIAM Journal on Scientific Computing, Vol. 27, No. 3 , 2005, pp. 873-892. doi: $10.1137 / 040613767$

[19] Elssel, K., and Voss, H., "An a Priori Bound for Automated Multilevel Substructuring," SIAM Journal on Matrix Analysis and Applications, Vol. 28, No. 2, 2007, pp. 386-397. doi: $10.1137 / 040616097$

[20] Jakobsson, H., and Larson, M. G., "A Posteriori Error Analysis of Component Mode Synthesis for the Elliptic Eigenvalue Problem," Computer Methods in Applied Mechanics and Engineering, Vol. 200, Nos. 41-44, 2011, pp. 2840-2847. doi:10.1016/j.cma.2011.05.002

[21] Park, K. C., Kim, J. G., and Lee, P. S., "A Mode Selection Criterion Based on Flexibility Approach in Component Mode Synthesis," 53th AIAA/ASME/ASCE/AHS/ASC Structures, Structural Dynamics, and Materials Conference, AIAA Paper 2012-1883, 2012.

[22] Markovic, D., Park, K. C., and Ibrahimbegovic, A., "Reduction of Substructural Interface Degrees of Freedom in Flexibility-Based Component Mode Synthesis," International Journal of Numerical Methods in Engineering, Vol. 70, No. 2, 2007, pp. 163-180. doi:10.1002/(ISSN)1097-0207

[23] Ibrahimbegovic, A., and Wilson, E. L., "Automated Truncation of Ritz Vector Basis in Modal Transformation," ASCE Journal of Engineering Mechanics Division, Vol. 116, No. 11, 1990, pp. 2506-2520. doi:10.1061/(ASCE)0733-9399(1990)116:11(2506)

[24] Kim, J. G., and Lee, P. S., "An Accurate Error Estimator for Guyan Reduction," Computer Methods in Applied Mechanics and Engineering, Vol. 278, Aug. 2014, 1-19. doi:10.1016/j.cma.2014.05.002

[25] Kim, J. G., Lee, K. H., and Lee, P. S., "Estimating Relative Eigenvalue Errors in the Craig-Bampton Method," Computers and Structures, Vol. 139, July 2014, pp. 54-64. doi:10.1016/j.compstruc.2014.04.008

[26] Dvorkin, E. N., and Bathe, K. J., "AContinuum Mechanics Based FourNode Shell Element for General Nonlinear Analysis," Engineering Computations, Vol. 1, No. 1, 1984, pp. 77-88. doi:10.1108/eb023562

[27] Jeon, H. M., Lee, P. S., and Bathe, K. J., "The MITC3 Shell Finite Element Enriched by Interpolation Covers," Computers and Structures, Vol. 134, April 2014, pp. 128-142. doi:10.1016/j.compstruc.2013.12.003 
[28] Lee, Y., Lee, P. S., and Bathe, K. J., "The MITC3+ Shell Finite Element and Its Performance," Computers and Structures, Vol. 138, July 2014, pp. 12-23.

doi:10.1016/j.compstruc.2014.02.005

[29] Lee, Y. G., Yoon, K. H., and Lee, P. S., "Improving the MITC3 Shell Finite Element by Using the Hellinger-Reissner Principle," Computers and Structures, Vols. 110-111, Nov. 2012, pp. 93-106. doi:10.1016/j.compstruc.2012.07.004

[30] Ibrahimbegovic, A., Chen, H. C., Wilson, E. L., and Taylor, R. L., "Ritz Method for Dynamic Analysis of Linear Systems with Non-
Proportional Damping," International Journal for Earthquake Engineering and Structural Dynamics, Vol. 19, No. 6, 1990, pp. 877889.

doi:10.1002/(ISSN)1096-9845

S. Griffin Associate Editor 